\title{
CONTRIBUIÇÕES DO ENFERMEIRO NO ENFRENTAMENTO DA HANSENÍASE NO BRASIL: REVISÃO DE ESCOPO
}

\author{
NURSES' CONTRIBUTIONS TO CONFRONTING LEPROSY IN BRAZIL: SCOPING REVIEW
}

\author{
CONTRIBUCIONES DE LAS ENFERMERAS PARA EL TRATAMIENTO DE LA LEPRA EN BRASIL: REVISIÓN \\ DE ALCANCE
}

\author{
Letycia Luciano Lucena Alves* ${ }^{*}$ (D) \\ Maressa Samai Pinheiro Silva Smith ${ }^{1}$ (D) \\ Camila Priscila Abdias do Nascimento ${ }^{2}$ (D)
}

\begin{abstract}
${ }^{1}$ Enfermeira graduada pela Universidade Potiguar (UnP), Natal, Rio Grande do Norte, Brasil; ${ }^{2}$ Enfermeira graduada pela (UFRN), Mestre e doutoranda em Enfermagem - Programa de Pós-Graduação em Enfermagem - PGENF da Universidade Federal do Rio Grande do Norte - UFRN Natal, Rio Grande do Norte, Brasil.*Autor correspondente: letycia.lucena@gmail.com.
\end{abstract}

Recebido: 28/09/2021 | Aprovado: 30/11/2021| Publicado: 14/12/2021

\begin{abstract}
Resumo: O objetivo do presente estudo é evidenciar estratégias utilizadas pelo enfermeiro no enfrentamento da hanseníase no Brasil. O estudo trata-se de uma revisão de escopo (Scoping Review), seguindo as orientações contidas no manual de revisões do Joanna Briggs Institute (JBI), de 2020. Realizada através de consulta online nas seguintes bases de dados: Scientific Eletronic Library Online (SciELO), Literatura Latino Americana e do Caribe em Ciências da Saúde (LILACS) e Biblioteca Virtual em Saúde do Ministério da Saúde (BVSMS). A amostra inicial foi constituída por 356 artigos, sendo utilizados um total de 22 estudos para elaboração desta revisão. É percebido que a hanseníase está fortemente associada aos determinantes e condicionantes de saúde, demonstrando a necessidade de uma abordagem mais ampla com a população conduzida pelo enfermeiro no contexto da atenção básica, por meio da educação em saúde em uma linguagem facilitada e efetiva, contribuindo para o estreitamento de vínculos entre a população e o profissional de modo a evitar o abandono ao tratamento. Medidas preventivas, de diagnóstico e tratamento da hanseníase ofertadas no cenário da atenção básica e, implementadas pelos profissionais enfermeiros possuem um alto grau de relevância para controle da doença no território, evitando que a patologia suscite incapacidades físicas que dificultem a socialização do portador de hanseníase em seu convívio social.
\end{abstract}

Palavras-chave: Hanseníase. Cuidados de Enfermagem. Atenção Primária. Prevenção.

Abstract: The purpose of this study is show strategies used by nurses in coping with leprosy in Brazil. The study is a scope review (Scoping Review), following the guidelines contained in the Joanna Briggs Institute (JBI) review manual, 2020. It was carried out though online consultation in the following databases: Scientific Eletronic Library Online (SciELO, Latin American and Caribbean Literature on Health Sciences (LILACS) and Virtual Health Library of the Ministry of Health (BVSMS). The initial sample consisted of 356 articles with a total of 22 studies being used to prepare this review. It is noticed that leprosy is strongly associated with health determinants and conditions, demonstrating the need for a broader approach with the population led by nurses in the context of primary care, though health education in a facilited and effective language, contributing to the strengthening of bonds between the population and the professional in order to avoid treatment abandonment. Preventive, diagnostic and treatment measures for leprosy offered in the primary care setting and implemented by professional nurses have a high degree of relevance for disease control in the territory, preventing the pathology from causing physical disabilities that hinder the socialization of leprosy patients in your social life.

Keywords: Leprosy. Nursing Care. Primary care. Prevention.

Resumen: El objetivo de este estudio es destacar las estrategias utilizadas por las enfermeras para hacer frente a la lepra en Brasil. El estudio es una revisión de alcance (Scoping Review), siguiendo los lineamientos contenidos en el manual de revisión del Joanna Briggs Institute (JBI) de 2020. Se realizó mediante consulta en Internet en las siguientes bases de datos: Scientific Electronic Library Online (SciELO), Latinoamérica y Literatura Caribeña en Ciencias de la Salud (LILACS) y Biblioteca Virtual en Salud del Ministerio de Salud (BVSMS). La muestra inicial consistió en 356 artículos, con un total de 22 estudios que se utilizaron para preparar esta revisión. Se advierte que la lepra está fuertemente asociada a determinantes 
y condicionantes de salud, evidenciando la necesidad de un abordaje más amplio con la población liderado por enfermeras en el contexto de la atención primaria, a través de la educación en salud en un lenguaje facilitado y eficaz, contribuyendo al fortalecimiento de los lazos entre la población y el profesional para evitar el abandono del tratamiento. Las medidas de prevención, diagnóstico y tratamiento de la lepra ofrecidas en el ámbito de la atención primaria y implementadas por enfermeras profesionales tienen un alto grado de relevancia para el control de la enfermedad en el territorio, evitando que la patología genere discapacidades físicas que dificulten la socialización de los leprosos en su vida social.

Palabras-clave: Lepra. Atención de Enfermería. Atención primaria. Prevención.

\section{INTRODUÇÃO}

A hanseníase é classificada como uma doença infectocontagiosa de evolução crônica, cujo agente etiológico, o bacilo Mycobacterium leprae, apresenta alta infectividade e baixa patogenicidade. Seu modo de transmissão ocorre por meio de gotículas ou aerossóis, e se manifesta através de sinais e sintomas dermatoneurológicos resultando em um comprometimento de pele, mucosas e sistema nervoso periférico, promovendo perdas axonais extensas se não tratada previamente. Considerada uma doença incapacitante e deformante independentemente de idade ou sexo, adultos e crianças podem ser acometidas (Araújo et al., 2017; Costa et al., 2019; Cortela et al., 2020).

Atualmente, a hanseníase é considerada como grave problema de saúde pública a nível mundial. No cenário epidemiológico, segundo a Organização Mundial de Saúde (OMS) (2020) foram notificados 202.185 novos casos de hanseníase em todo o mundo, sendo a Índia o país a manifestar maior carga da doença contabilizando (120.334 casos), seguidos do Brasil (27.864) e Indonésia (17.017), respectivamente. Do montante identificado em território brasileiro, este distribui-se entre as regiões do país, retratando a detecção de novos casos principalmente nos estados do Mato Grosso (4.186), Maranhão (2.573), Pará (1.983), Pernambuco (1.806), Bahia (1.436), Tocantins (1.353) e Goiás (1.180) (Brasil, 2021).

À vista disso, o Ministério da Saúde elaborou a Estratégia Nacional de Enfrentamento da Hanseníase 2019-2022, com o objetivo de reduzir a carga da doença em âmbito nacional até o fim de 2022. Contemplando metas como fortalecimento da gestão do programa, enfrentamento da hanseníase e suas complicações, além de, implantar em todos as capitais brasileiras, canais para registrar práticas de discriminação aos portadores de hanseníase e seus familiares (Brasil, 2021).

É válido ressaltar que, a hanseníase faz parte da Lista Nacional de Notificação Compulsória de Doenças, Agravos e Eventos de Saúde do Brasil. Desse modo, os profissionais de saúde possuem a obrigatoriedade de notificar os casos da doença ao Sistema de Informação de Agravos de Notificação (SINAN). Esse registro auxilia na análise das informações registradas no sistema, a fim de, detectar as áreas que apresentam maior vulnerabilidade à doença no país, bem como, identificar fragilidades na qualidade da vigilância, para posteriormente definir estratégias de enfrentamento (Souza, Magalhães \& Luna, 2020; Brasil, 2021).

Palmeira et al. (2020) destaca que, um dos principais acometimentos dessa patologia está relacionado as incapacidades físicas. Na qual poderá progredir a ponto de gerar deformidades que comprometem e interferem 
na qualidade de vida do indivíduo. Além do impacto físico causado nos portadores de hanseníase, a doença implica similarmente em aspectos psicossociais, decorrentes principalmente do diagnóstico realizado tardiamente.

Por meio da análise epidemiológica da Secretaria de Vigilância em Saúde, foi observado que a proporção de casos novos em relação aos modos de encaminhamento e demanda espontânea foram os que obtiveram maior frequência, registrando $84 \%$. Do total de $81,9 \%$ dos casos avaliados, 36,8\% manifestaram algum grau de deformação. Quanto a proporção de cura de casos novos diagnosticados, o Brasil apresentou redução de 79,4\%, valor considerado regular, de acordo com a estratégia estabelecida de combate a hanseníase. Mediante os dados apresentandos, torna-se imprescindível a participação do enfermeiro através da implementação de ações de enfrentamento à doença (Brasil, 2021).

Na conjuntura da Atenção Primária à Saúde (APS), as atribuições exercidas pelo enfermeiro estão ancoradas na participação fundamental como integrante da equipe de saúde, assumindo um papel de relevância e proatividade nas ações de busca, prevenção, promoção e vigilância no contexto da hanseníase. Colaborando com o tratamento por meio das consultas de enfermagem, solicitando exames complementares, além de articular e coordenar ações desenvolvidas na APS (Ribeiro et al., 2017; Sousa, Silva \& Xavier, 2017).

Nesse contexto, Morais \& Furtado (2018) afirmam que é fundamental a realização do diagnóstico através do exame clínico, quando se busca sinais dermatoneurológicos por meio do manuseio de um conjunto de monofilamentos de Semmes-Weinstein em pontos específicos das mãos e dos pés para avaliação da sensibilidade, por exemplo. Dessa forma, torna-se essencial que ocorra a investigação do grau de incapacidade física dos pacientes portadores de hanseníase na ocasião do diagnóstico.

Nesse cenário, é importante que enquanto profissional de saúde o enfermeiro esteja envolvido em ações promocionais, preventivas e curativas relacionadas a hanseníase. Para tanto, é necessário que o cuidado também esteja voltado para a educação permanente desses profissionais, visto que, o despreparo destes, atrasam o diagnóstico precoce impactando diretamente na evolução da doença. Ademais, é importante desenvolver aspectos educacionais à população com o propósito de conhecer os sinais e sintomas para que mediante um sinal clínico, os pacientes possam buscar uma unidade de saúde com maior antecedência atenuando o desenvolvimento da doença (Cavalcante, Lorocca \& Chaves, 2020).

Mediante a isso, a Política Nacional de Educação Permanente em Saúde (PNEPS), capacita os profissionais de saúde para a melhoria da qualidade assistencial das ações e serviços de saúde oferecidos pelo Sistema Único de Saúde (SUS). Assim, a educação permanente é primordial e se exprime na necessidade de estar inserida no cotidiano do profissional de saúde. No entanto, ainda há desafios importantes pautados no reflexo negativo dos trabalhadores que ainda reproduzem o estigma social da doença, refletindo na efetividade das orientações a esses pacientes (Carvalho et al., 2018; Moreira et al., 2021).

A educação permanente objetiva proporcionar melhorias na qualidade de ações relacionadas ao controle da hanseníase. Todavia, ainda é percebido que há insuficiências em suas estratégias quanto a confiança 
profissional no diagnóstico da doença e ao manejo da pessoa hansênica. Com isso, apenas a disseminação de informações sem um processo coletivo de ensino-aprendizagem, não é suficiente para o enfrentamento dessa questão no cotidiano desses profissionais (Ribeiro et al., 2017; Carvalho et al., 2018; Moreira et al., 2021).

Diante da relevância da atuação do profissional enfermeiro na promoção do cuidado ao paciente portador de hanseníase na atenção primária e o atual panorama em relação a evolução da patologia em território brasileiro, este estudo apresenta como objetivo evidenciar estratégias utilizadas pelo enfermeiro no enfrentamento da hanseníase no Brasil. Tal escolha justifica-se pelas lacunas identificadas na educação permanente destes profissionais consolidando uma ineficiência assistencial aos portadores de hanseníase.

\section{MATERIAL E MÉTODOS}

Este estudo trata-se de uma revisão de escopo (Scoping Review), e apresenta como finalidade mapear os principais conceitos que fundamentam a pesquisa, bem como, averiguar a extensão e natureza das produções, selecionar estudos, extrair e sumarizar os dados, além de realizar o apontamento de lacunas existentes na literatura e a difusão dos dados da investigação (Cordeiro \& Soares, 2019; Joanna Briggs Institute, 2020).

A elaboração da presente pesquisa foi realizada seguindo as orientações contidas no manual de revisões do Joanna Briggs Institute (JBI), de 2020. Posteriormente, foi iniciada a elaboração do protocolo de revisão de escopo e o diagrama de fluxo, instruído por meio do Preferred Reporting Items for Systematic reviews and MetaAnalyses extension for Scoping Reviews (PRISMA-ScR). Salienta-se ainda que o estudo em questão foi registrado na plataforma Open Science Framework (OSF) (https://osf.io/ajtyf/) (Joanna Briggs Institute, 2020).

A formulação da questão norteadora foi baseada na estratégia População, Conceito e Contexto (PCC) recomendado pelo JBI. Dessa forma, foi estabelecido: P - os profissionais enfermeiros; C - as contribuições ofertadas mediante o enfrentamento da hanseníase; C - no Brasil. Assim, com base na estratégia mnemônica supracitada, foi determinada a seguinte questão de pesquisa: Quais as contribuições assistenciais do profissional enfermeiro ao portador de hanseníase diante do aumento da incidência de casos no Brasil?

Para produção do estudo, a pesquisa foi realizada por meio de consulta online ao decorrer dos meses de abril a novembro de 2021, nas seguintes bases de dados científicas: Scientific Eletronic Library Online (SciELO), Literatura Latino Americana e do Caribe em Ciências da Saúde (LILACS) e Biblioteca Virtual em Saúde do Ministério da Saúde (BVSMS). Os Descritores em Ciência da Saúde (DeCS) utilizados para seleção dos artigos foram: "hanseníase", "cuidados de enfermagem", “atenção primária”, "prevenção". Utilizando o operador boleano $A N D$ para combinação desses.

Tabela 1 - Sintaxe de busca da temática com o uso de descritores nas bases de dados científicas. Natal, Rio Grande do Norte. Brasil, 2021.

\begin{tabular}{cc}
\hline Fontes de Dados & Sintaxe de Busca \\
\hline SciELO & "hanseníase" and "enfermagem"
\end{tabular}




\begin{tabular}{cc} 
LILACS & $\begin{array}{c}\text { "cuidados de enfermagem" and "hanseníase" } \\
\text { "enfermagem" and "prevenção" and "hanseníase" }\end{array}$ \\
\hline BVSMS & Fonte: Elaboração dos autores,2021.
\end{tabular}

Os estudos foram selecionados de forma independente por dois pesquisadores da equipe, e nos casos em que houve conflitos, um terceiro pesquisador teve o poder da tomada de decisão na ausência de consenso. Foram estabelecidos como critérios de inclusão artigos disponíveis na íntegra e gratuitamente, publicados entre os anos de 2017 a outubro de 2021, produzidos nas línguas portuguesa, inglesa e espanhola.

As etapas das estratégias de busca dividiram-se em três passos: no primeiro utilizou-se os descritores em saúde adequados as bases de dados consultadas (SciELO, LILACS e BVSMS) resultando em um apanhado de 356 artigos. Na segunda parte após leitura dos títulos e resumos com objetivo de selecionar estudos pertinentes a questão norteadora da pesquisa, resultou na manutenção de 28 artigos. E a terceira etapa consistiu após a leitura dos estudos em sua integralidade, por meio da coleta de informações selecionadas quanto a forma do estudo: autor (es), o ano de publicação, tipo de estudo e objetivo da pesquisa, totalizando 22 artigos para construção deste estudo. Seguindo com o agrupamento e apresentação em carácter descritivo, por meio de tabelas de modo a propiciar uma melhor visualização dos resultados obtidos.

\section{RESULTADOS}

Conforme a busca nas bases de dados científicas, foram identificados 22 estudos em consonância com os critérios de elegibilidade da metodologia após leitura integral dos artigos selecionados, conforme demonstrado na figura 1.

Figura 1 - Fluxograma do processo de seleção dos estudos adaptado do PRISMA- ScR. Natal, Rio Grande do Norte.
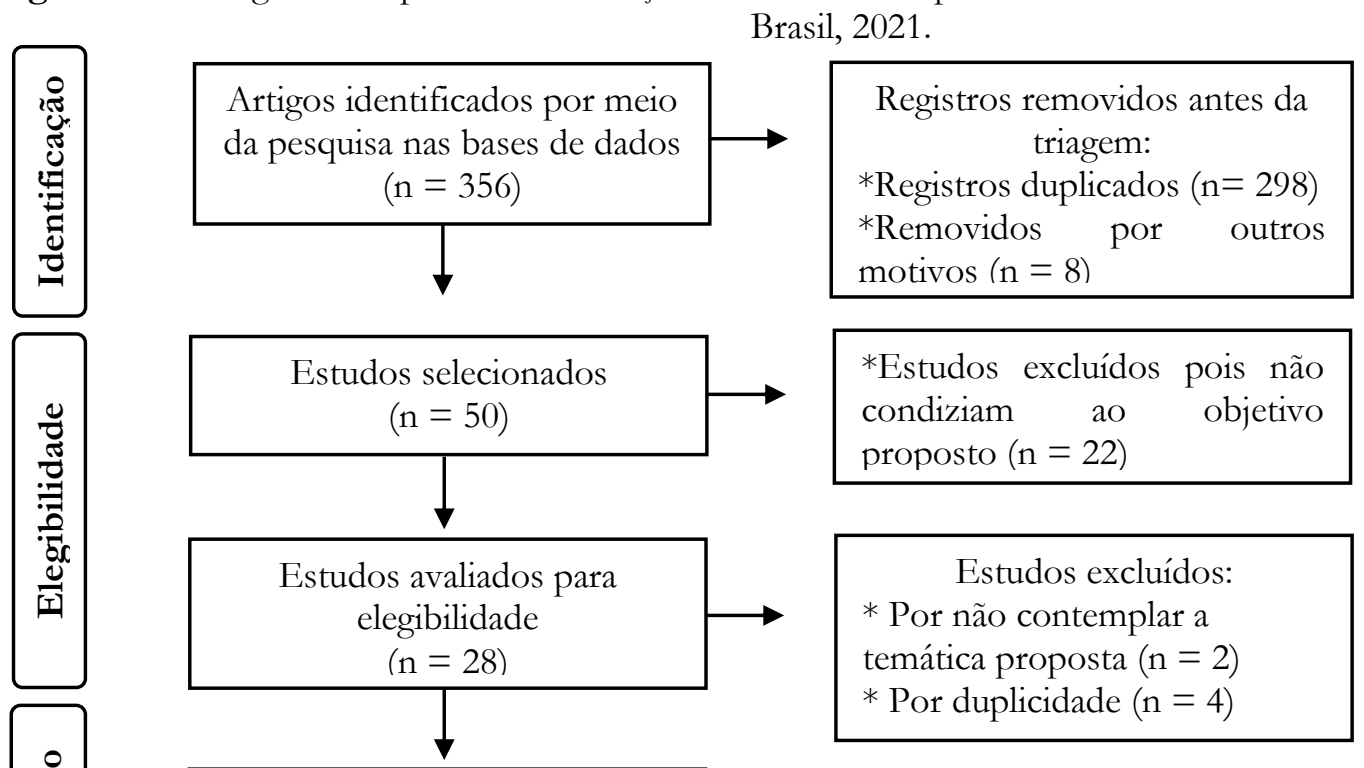

Estudos selecionados para a revisão $(\mathrm{n}=22)$ 
Fonte: Elaboração dos autores, 2021.

A sumarização dos estudos foi elaborada de forma descritiva, permitindo a organização e agrupamento das informações com o auxílio de um quadro elaborado pelos autores. As evidências foram desenvolvidas em sua maioria como estudos qualitativos, seguidos de quantitativos, revisão integrativa, estudo ecológico e relato de experiência, respectivamente.

Quadro 1 - Quadro com amostra dos resultados elencados para pesquisa. Natal, Rio Grande do Norte. Brasil, 2021.

$\begin{array}{ccccc}\text { Autor } & \text { Título } & \text { Principais Resultados } & \text { Tipo de } & \text { Nome do } \\ \text { (Ano/ País) } & & & \text { Estudo } & \text { Periódico }\end{array}$

\begin{tabular}{|c|c|c|c|c|}
\hline $\begin{array}{l}\text { Araújo et al. } \\
\text { 2017/Brasil }\end{array}$ & $\begin{array}{c}\text { Tendência de } \\
\text { indicadores } \\
\text { epidemiológicos } \\
\text { da hanseníase em } \\
\text { um estado } \\
\text { endêmico }\end{array}$ & $\begin{array}{c}\text { Foram analisados } 12.134 \text { casos } \\
\text { novos de hanseníase. Observou- } \\
\text { se tendência decrescente para } \\
\text { taxa de detecção geral de }-4,8 \% \\
\text { e para taxa de detecção em } \\
\text { menores de } 15 \text { anos de }-6,7 \% \text {. A } \\
\text { proporção de casos novos de } \\
\text { hanseníase com grau } 2 \text { de } \\
\text { incapacidade física no } \\
\text { diagnóstico apresentou } \\
\text { tendência estacionária de } \\
2,7 \% \text {. }\end{array}$ & $\begin{array}{c}\text { Estudo } \\
\text { ecológico de } \\
\text { tendência } \\
\text { temporal. }\end{array}$ & $\begin{array}{c}\text { Revista da Rede } \\
\text { de Enfermagem } \\
\text { do Nordeste }\end{array}$ \\
\hline $\begin{array}{l}\text { Araújo \& Silva } \\
\text { 2019/Brasil }\end{array}$ & $\begin{array}{l}\text { Vulnerabilidade } \\
\text { em casos de } \\
\text { hanseníase na } \\
\text { atenção primária } \\
\text { à saúde }\end{array}$ & $\begin{array}{l}\text { Apresentou a vulnerabilidade } \\
\text { dos pacientes portadores de } \\
\text { hanseníase de forma ampla, e } \\
\text { relacionou as incapacidades } \\
\text { físicas com baixas condições } \\
\text { socioeconômicas e sexo } \\
\text { masculino, dentre outros. }\end{array}$ & $\begin{array}{l}\text { Revisão } \\
\text { narrativa. }\end{array}$ & $\begin{array}{c}\text { Revista } \\
\text { Científica da } \\
\text { Escola Estadual } \\
\text { de Saúde } \\
\text { Pública de } \\
\text { Goiás }\end{array}$ \\
\hline $\begin{array}{l}\text { Cavalcante, } \\
\text { Lorocca \& } \\
\text { Chaves } \\
\text { 2020/Brasil }\end{array}$ & $\begin{array}{l}\text { Múltiplas } \\
\text { dimensões da } \\
\text { gestão do } \\
\text { cuidado à } \\
\text { hanseníase e os } \\
\text { desafios para a } \\
\text { eliminação }\end{array}$ & $\begin{array}{l}\text { Evidenciou-se contradições nas } \\
\text { múltiplas dimensões da Gestão } \\
\text { do Cuidado, permitindo } \\
\text { compreender que os desafios } \\
\text { para o enfrentamento da } \\
\text { hanseníase não se encontram } \\
\text { apenas no âmbito biológico, mas } \\
\text { na sua determinação social. }\end{array}$ & $\begin{array}{l}\text { Estudo de caso } \\
\text { único, de cunho } \\
\text { exploratório e } \\
\text { abordagem com } \\
\text { métodos mistos. }\end{array}$ & $\begin{array}{c}\text { Revista da } \\
\text { Escola de } \\
\text { Enfermagem da } \\
\text { USP }\end{array}$ \\
\hline $\begin{array}{l}\text { Cortela et al. } \\
\text { 2020/Brasil }\end{array}$ & $\begin{array}{c}\text { Aceitabilidade da } \\
\text { quimioprofilaxia } \\
\text { em área endêmica } \\
\text { para hanseníase: } \\
\text { projeto PEP- } \\
\text { Hans Brasil }\end{array}$ & $\begin{array}{l}\text { A compreensão se mostrou } \\
\text { relacionada ao cuidado da } \\
\text { equipe de saúde. Aceitar ou não } \\
\text { a medicação revelou-se } \\
\text { relacionada ao medo, confiança } \\
\text { e proteção, operacionalidade da } \\
\text { estratégia, autoestima e } \\
\text { insegurança quanto à } \\
\text { intervenção. }\end{array}$ & $\begin{array}{c}\text { Estudo } \\
\text { qualitativo. }\end{array}$ & $\begin{array}{c}\text { Revista } \\
\text { Cadernos de } \\
\text { Saúde Pública }\end{array}$ \\
\hline $\begin{array}{l}\text { Costa et al. } \\
\text { 2019/Brasil }\end{array}$ & $\begin{array}{l}\text { Aspectos clínicos } \\
\text { e epidemiológicos } \\
\text { da hanseníase }\end{array}$ & $\begin{array}{l}\text { Registraram-se } 35.853 \text { casos de } \\
\text { hanseníase, sendo a maioria do } \\
\text { sexo masculino na faixa etária }\end{array}$ & $\begin{array}{l}\text { Estudo } \\
\text { quantitativo, } \\
\text { descritivo, }\end{array}$ & $\begin{array}{l}\text { Revista de } \\
\text { Enfermagem } \\
\text { UFPE online }\end{array}$ \\
\hline
\end{tabular}


entre 40-59 anos, de cor parda, com nível fundamental incompleto e residente em zona urbana.

Façanha et al. 2020/ Brasil

Freitas et al. 2019/Brasil

Mascarenhas et al. 2021/ Brasil

Monteiro et al. 2018/Brasil

\section{Morais \& \\ Furtado 2018/Brasil}

\begin{abstract}
Análise das incapacidades físicas por hanseníase em uma cidade do interior do
\end{abstract} Maranhão, Brasil

Percepção de adolescentes sobre hanseníase

A importância das ações realizadas pelo enfermeiro no controle da hanseníase: revisão integrativa

$$
\begin{aligned}
& \text { Educação em } \\
& \text { saúde para } \\
& \text { hanseníase: } \\
& \text { experiência da } \\
& \text { enfermagem }
\end{aligned}
$$

\begin{abstract}
A hanseníase atinge, principalmente, indivíduos de cor parda, do sexo masculino e com uma mediana de idade de 50 anos. Há o predomínio do grau 0 de incapacidade física, das

formas clínicas dimorfa e virchowiana, indicando a detecção tardia da doença.
\end{abstract}
Verifica-se que nas falas dos adolescentes, há pouco conhecimento sobre a

hanseníase, sem discernimento sobre os aspectos gerais da doença, associando-a a outras enfermidades.

Afirma-se sobre a importância das ações realizadas pelos enfermeiros no controle da hanseníase, sendo de fundamental relevância a identificação precoce, o acompanhamento dos casos e, sobretudo, a quebra do estigma social ainda presente na sociedade.

A educação em saúde, quando entendida pelo público-alvo contribui no desenvolvimento do diagnóstico precoce da hanseníase, bem como auxilia na prevenção de incapacidades trazidas pela doença

Essa prática apresenta como percussor principal o enfermeiro, profissional que busca o atendimento integral do indivíduo, por meio da promoção e prevenção da saúde.

Constatou-se que pacientes do sexo masculino com baixa escolaridade, forma clínica dimorfa, virchowiana e neural pura, classificação operacional multibacilar e a presença de um ou mais nervos afetados tiveram maior chance de apresentar algum grau de incapacidade

transversal.

Pesquisa epidemiológica, do tipo coorte retrospectiva, de base documental com abordagem quantitativa.

Pesquisa
qualitativa e
descritiva.

Revisão integrativa.

Estudo descritivo de abordagem qualitativa, do tipo relato de experiência.
Revista Saúde (Santa Maria)

$$
\begin{gathered}
\text { Research, } \\
\text { Society and } \\
\text { Development. }
\end{gathered}
$$

\section{Revista de \\ Casos e}

Consultoria

$\begin{array}{cc}\text { Estudo } & \text { Revista de } \\ \text { quantitativo, } & \text { Enfermagem } \\ \text { transversal, } & \text { UFPE online } \\ \text { retrospectivo. } & \end{array}$


física.
Moreira et al. 2021/ Brasil

Oliveira et al. 2020a/ Brasil

Oliveira et al. 2020b/Brasil

Palmeira et al. 2020/Brasil

Pinheiro et al. 2017/Brasil
Atuação dos enfermeiros nas ações de controle da hanseníase na atenção primária à saúde: revisão integrativa

Análise epidemiológica da hanseníase por sexo na Paraíba.

Diagnósticos de enfermagem em pessoas com hanseníase: aproximação entre teoria de Orem e a CIPE

\section{Percepção de} pacientes com hanseníase sobre suas necessidades humanas básicas alteradas: indícios para o autocuidado

Compreendendo a "alta em hanseníase": uma análise de conceito
Foram selecionados 12 artigos para o estudo, ao analisar constatou-se a importância de integrar as ações de hanseníase na Atenção Primária à Saúde, visto que, apesar de algumas fragilidades, o serviço é capaz de realizar as ações de controle da hanseníase.

Analisou-se 6.271 casos novos de hanseníase, com maior incidência no

sexo masculino. A hanseníase acomete ambos os sexos, no entanto, quando comparados às mulheres, os homens apresentam as formas mais graves da doença; classificação multibacilar (66.79\%), forma clínica dimorfa (36.14\%) e grau 2 de incapacidade física $(11.66 \%)(\mathrm{p}<00,1)$.

O processo de elaboração dos diagnósticos de enfermagem foi apoiado em raciocínio clínico, no modelo de sete eixos da CIPE® e na ISO 18.104. As inferências diagnósticas foram validadas por três juízes.

Foram detectadas cinco necessidades alteradas nec essidades fisiológicas, de segurança, de amor e/ou sociais, de estima e de autorrealização. Evidenciou-se que percepção das necessidades alteradas estimula ações de autocuidado, com vistas a sua satisfação.

Identificou-se alta por cura, alta medicamentosa, alta bacteriológica e pós-alta como possíveis usos do conceito. Os atributos definidos foram conclusão da poliquimioterapia, conclusão da poliquimioterapia para paucibacilares, conclusão da poliquimioterapia para multibacilares e cura da hanseníase.

$\begin{array}{cc}\text { Revisão } & \text { Diversitas } \\ \text { integrativa. } & \text { Journal }\end{array}$

Pesquisa

Research, documental, Society and descritiva, de Development. série temporal, com abordagem quantitativa

Estudo transversal, descritivo.

Revista de Enfermagem UFPE online

Pesquisa qualitativa e descritiva.

Estudo teórico Revista Gaúcha pautado no referencial metodológico de análise de conceito.

\section{Revista de \\ Pesquisa \\ Cuidado é \\ Fundamental Online}


Ramos, J. S., Costa, L. R. \& Santos, W. L. 2019/ Brasil

Ribeiro et al. 2017/Brasil

Sousa, Silva \& Xavier 2017/Brasil

Souza, Magalhães \& Luna 2020/Brasil

Souza, Lanza \&
Souza
2018/Brasil

Vieira, Martínez-Rieira \& Lana, 2020/Brasil
Dificuldades da enfermagem no manejo da hanseníase na atenção primária

A visão do
profissional
enfermeiro sobre
o tratamento da
hanseníase na
atenção básica
Atributos da

Atenção Primária em Saúde no controle da hanseníase: ótica do enfermeiro

Hanseníase e carência social: definição de áreas prioritárias em estado endêmico do Nordeste brasileiro

Sensibilização dos Agentes

Comunitários de Saúde para a atuação nas ações prevenção e controle da hanseníase: relato de experiência

Qualidade da atenção primária e os efeitos em indicadores de monitoramento da hanseníase
Os resultados demonstraram que os anos que mais ocorreram publicações foram 2010 e 2018, com quatro publicações respectivamente. Constatou-se que o conhecimento sobre a doença mostra-se fragilizado, impactando diretamente no diagnóstico e no tratamento.

A supervisão está relacionada ao tratamento correto, garantia de cura, redução de sequelas e interrupção da transmissão da doença.

O município apresentou, pela média dos escores gerais e dos atributos essenciais e derivados, alta orientação para as ações de controle da hanseníase, com exceção do atributo acesso, que recebeu média abaixo do ponto de corte.

Dos municípios, $60,4 \%$ ( $\mathrm{n}=$ 252) apresentaram muito baixa condição de vida. Observou-se associação entre as condições de vida e a detecção da hanseníase, com maiores coeficientes no grupo de município com melhor condição de vida $(p<0,001)$.

Foi elaborado um plano de mobilização da comunidade sobre hanseníase. E partir da sensibilização da comunidade na igreja, houve a demanda espontânea ao serviço de saúde de uma pessoa com sinais dermatológicos, sendo que o diagnóstico de hanseníase foi confirmado em março de 2016.

Houve correlação entre escore geral e percentual de casos tratados na atenção primária e de cobertura de equipe de saúde da família. No escore derivado houve correlação com percentual de casos tratados na atenção primária e no essencial, com a proporção e taxa de diagnósticos com grau 2 de incapacidade física.

\author{
Pesquisa \\ integrativa. \\ Revista JRG de \\ Estudos \\ Acadêmicos
}

Pesquisa do tipo

Revista descritiva, com abordagem qualitativa.

Brasileira em Promoção da Saúde

Estudo de Revista Baiana avaliação da qualidade em de Enfermagem saúde.

\title{
Estudo ecológico.
}

Revista Brasileira de Epidemiologia

Relato de experiência.

HU Revista

Estudo transversal.
Revista

Brasileira de Enfermagem 


\section{DISCUSSÃO}

Para refletir sobre as contribuições do enfermeiro perante o enfrentamento da hanseníase, foi imprescindível realizar o mapeamento da literatura por meio da revisão de escopo, a fim de esclarecer e pontuar as ações desenvolvidas pelos enfermeiros frente aos obstáculos e manejo da hanseníase. Mediante os resultados encontrados, foi possível identificar que os estudos mais recentes sobre o tema podem estar relacionados com a incidência dos casos de hanseníase, visto que a doença é considerada uma questão de saúde pública e que o território brasileiro apresenta um elevado número de notificações efetivadas (Brasil, 2021).

Nessa conjuntura, o enfermeiro participa ativamente do processo de controle da hanseníase. Para tanto, foi evidenciado que as consultas de enfermagem se enquadram em um espaço fundamental para ações de prevenção dessa enfermidade, proporcionando um vínculo com o paciente pautado na confiança $\mathrm{e}$ humanização. É onde o enfermeiro realiza avaliações minuciosas e identificação de sinais e sintomas sugestivos a hanseníase ou aponta complicações manifestadas pela doença, possibilitando o diagnóstico precoce, aumentando suas chances de cura (Monteiro et al., 2018; Ramos, Costa \& Santos, 2019; Mascarenhas et al., 2021).

Com isso, é importante ressaltar que o seu tratamento possui cobertura completa das políticas públicas de saúde. Os enfermeiros que atuam com esse público, acompanham com afinco os pacientes que se encontram em tratamento. Atentando para estratégias direcionadas com adesão ao regime terapêutico, no intuito de garantir o uso correto da medicação, atenuando as chances de abandono do tratamento, uma vez que as medicações causam reações hansênicas, e por vezes o paciente acredita que está declinando a uma piora (Ramos, Costa \& Santos, 2019; Cortela et al., 2020; Moreira et al., 2021).

Esse profissional, zela ainda pelo autocuidado do paciente, supervisionando e orientando-o quanto aos cuidados com a pele, na assistência com curativos e retirada de calosidades, por exemplo. Ademais, a fim de conter a doença o profissional ainda examina os contactantes de pacientes infectados, por meio da vigilância de contatos, o que confere uma ferramenta importante para profilaxia e favorece o diagnóstico precoce. Além disso, o enfermeiro contribui no gerenciamento de atividades cujo objetivo do desenvolvimento vise o combate a hanseníase e viabilize a diminuição de casos da doença (Ribeiro et al., 2017; Ramos, Costa \& Santos, 2019).

O Boletim Epidemiológico de Hanseníase 2021 do Ministério da Saúde (MS) aponta que nos anos de 2015 a 2019 foram notificados 137.380 novos casos da doença no Brasil segundo sexo e faixa etária. Em relação ao sexo $(\mathrm{m}=$ masculino $)$ e $(\mathrm{f}=\mathrm{feminino})$ bem como o número de casos anuais registrados, verifica-se no período de $2015(\mathrm{~m}=16.053 ; \mathrm{f}=12.708), 2016(\mathrm{~m}=14.062 ; \mathrm{f}=11.156), 2017(\mathrm{~m}=14.896 ; \mathrm{f}=11.981), 2018(\mathrm{~m}=15.582$; $\mathrm{f}=13.078)$ e $2019(\mathrm{~m}=15.394$; f 12.470) novos casos. Sendo observado maior prevalência na população masculina com 55,3\% no acumulado deste período, comparado a população feminina apresentando índice de $44,7 \%$. 
Por vezes, esse fator pode ser justificado pelo elevado contato social entre indivíduos do sexo masculino e sua constante exposição a fatores desencadeantes da doença, contribuindo para maior incidência de casos nesse grupo. Estudos afirmam que a heterogeneidade na incidência em ambos os sexos pode ser estimulada pelas diferenças no comportamento destes. Uma vez que os homens demonstram menos importância no que diz respeito a saúde e estética corporal, diferentemente das mulheres, que buscam serviços de saúde com maior frequência e realizam exames dermatológicos e rotineiros com menor periodicidade (Costa et al., 2019; Façanha et al., 2020; Oliveira et al., 2020a).

Conforme os dados do SINAN registrados entre os anos de 2015 a 2019, além de, manifestar-se predominante em indivíduos do sexo masculino, estes, apresentam ainda características como baixa escolaridade, categorizando cerca de 43,3\% de indivíduos com ensino fundamental incompleto, na faixa etária média de 70 a $79 \operatorname{anos}(61,2 \%)$, destacando-se com maior frequência na população parda à nível de território nacional (58,7\%), com relevância no percentual das regiões Nordeste (58.901 casos), Centro-Oeste (28.889) e Norte (26.505). Seguido dos brancos residentes nas regiões Sudeste que incluem (7.940) casos e o Sul (2.962), respectivamente (Brasil, 2021).

Essa heterogeneidade na distribuição de casos, pode também apresentar importante relação com os determinantes sociais da hanseníase a depender da localidade. Este, constitui um papel importante no processo de adoecimento da população, uma vez que, indivíduos inseridos em um contexto desfavorável, em situação que envolve habitação e alimentação precárias, apresentam maior susceptibilidade à doença. Conforme o cenário citado e com base nas evidências analisadas é notório que ainda há percalços na atenção à temática (Brasil, 2021).

As lacunas podem ser evidenciadas na carência da educação em saúde para a identificação precoce da patologia pelo usuário, o que interfere no reconhecimento de sinais e sintomas pela comunidade, bem como na deficiência do aprimoramento sobre a doença pelos profissionais de saúde, que por vezes não realiza o manejo adequado ao público. Assim, deve-se obter um trabalho profissional com o paciente seja individual ou coletivamente, o que pode interferir positivamente seja na prevenção, diagnóstico ou tratamento da doença (Pinheiro et al., 2017; Gomes et al., 2021).

Nessa conjuntura, é necessário que o profissional enfermeiro detenha conhecimento clínico e epidemiológico sobre a patologia para auxílio no diagnóstico, bem como, para contribuição na terapêutica aplicada, objetivando uma assistência de qualidade ao indivíduo. Impedindo a evolução da doença para graus incapacitantes, contando com estratégias que visem a prevenção da doença e a aderência fiel ao tratamento. Inserindo políticas de saúde específicas voltadas para o público do sexo masculino e a promoção de ações de avaliação e educação em saúde acolhedoras relacionadas a hanseníase, por exemplo. (Pinheiro et al., 2017; Façanha et al., 2019).

Em estudo realizado no município de Goiânia-GO, a respeito dos desvios de saúde pertinentes aos diagnósticos de enfermagem do portador de hanseníase, evidencia-se que a adesão ineficaz ao tratamento, percepção sensorial prejudicada, déficit de conhecimento sobre a doença, integridade da pele prejudicada e risco 
de queda, são uns dos principais fatores identificados que necessitam de intervenções de enfermagem, organizadas e baseadas no sistema de apoio e educação as necessidades desse paciente (Oliveira et al., 2020b).

Com o propósito de atenuar tais danos, é essencial que o enfermeiro realize ações de prevenção de incapacidades e promoção a saúde a esses indivíduos, investindo em planejamento terapêutico singular e na execução de estratégias traçadas. Aplicando ferramentas que o auxiliem na transmissão de informações sobre a doença. Informando aos familiares a respeito do compromisso no processo saúde doença de seu familiar portador da hanseníase, a fim de desmistificar o contágio, para que o familiar não se sinta sozinho nesse processo, de modo a favorecer a otimização do autocuidado e de transformações nas atitudes relacionadas a prevenção de incapacidades, bem como o apoio psicoemocional (Ribeiro et al., 2017; Oliveira et al., 2020b).

Souza, Lanza \& Souza (2018) salientam que, a hanseníase é uma doença que tem cura e seu tratamento é ofertado de forma gratuita por meio do SUS. E diante da importância de uma assistência integral e propícia ao paciente, no âmbito da atenção primária, podemos utilizar como estratégias ações educativas, educação permanente em saúde, sendo essas ações altamente relevantes para redução da carga da doença. Além de sensibilizar os agentes comunitários de saúde, para que auxiliem e abordem sobre a doença em suas visitas domiciliares, por exemplo.

Todavia, um dos principais obstáculos relacionados ao controle da hanseníase, ainda consiste na não adesão ao tratamento farmacológico prescrito ao paciente, que pode ocasionar o desenvolvimento de resistência aos fármacos, incapacidades físicas, além da permanência da cadeia de transmissão da doença. Desse modo, torna-se um grande desafio alcançar a integralidade da assistência a esta população, de modo a abordar tanto a promoção da saúde como a prevenção de agravos (World Health Organization, 2019; Oliveira et al., 2020a).

Nesse contexto, visto que a maioria dos pacientes ao perceberem o surgimento dos primeiros sinais e sintomas se direcionam às Unidades Básicas de Saúde (UBS), o enfermeiro deve estar preparado para orientá-los. À vista disso, é interessante que ao atuarem com indivíduos portadores de hanseníase, os enfermeiros invistam em um planejamento com execução estratégica direcionada a adesão do tratamento terapêutico assegurando a compreensão sobre ela e sua detecção precoce, favorecendo cuidados com a saúde do usuário da unidade (Monteiro et al., 2018; Araújo \& Silva, 2019).

A fim de proporcionar a construção do conhecimento e a emancipação da saúde, o enfermeiro que realiza ações de cunho educativo assegura não somente a transmissão de informações, mas também o aperfeiçoamento de comportamento e valores de modo criativo e participativo, contribuindo para autonomia do indivíduo. Com isso, terá como desafio identificar se as informações transmitidas ao seu público estão adequadas a ele, de modo a o estimular positivamente a incorporar essas informações em sua vida diária (Monteiro et al., 2018; Freitas et al., 2019).

Considerando este panorama, Palmeira et al. (2020) reconhece que é possível constatar que a hanseníase também pode suscitar múltiplos sentimentos que afetam negativamente a qualidade de vida dos pacientes, pois, podem causar alterações em suas necessidades humanas básicas englobando as dimensões fisiológicas, de estima, 
segurança, necessidades sociais, autorrelização e até mesmo de autoconfiança. No entanto, para que tais fatores sejam mitigados, torna-se indispensável a participação de uma equipe multidisciplinar que contemple diretrizes de cunho educacionais de forma horizontal e participativa, encorajando a prática do autocuidado a este público, priorizando uma escuta qualificada que viabilize ao paciente expor suas subjetividades baseado em suas dúvidas e anseios.

Vieira, Martínez-Rieira \& Lana (2020) reitera que tal achado corrobora com o índice de qualidade das ações de controle da hanseníase desenvolvidas pelos profissionais inseridos na atenção primária a saúde, onde reflete nos indicadores de monitoramento da doença. Além da importância da atuação da vigilância em saúde por meio da ação de práticas individualizadas e coletivas de acordo com as necessidades identificadas, que contemplem a discussão dos determinantes sociais em saúde, controle de riscos, bem como o planejamento de intervenções em conformidade com a realidade do território. Pois, destaca-se que a atenção primária a saúde detém o potencial de prestar uma assistência qualificada na condição em que o paciente está incluído.

Têm-se como limitação deste estudo, a escassez de trabalhos atualizados que abordem a temática proposta, contemplando também vertentes da prática em apoio com outros profissionais que fazem parte da rede de atenção. Portanto, reforça-se a orientação de construção de novos estudos que abordem ações conjuntas entre os profissionais inseridos na assistência à esta comorbidade.

\section{CONSIDERAÇÕES FINAIS}

Medidas preventivas, de diagnóstico e tratamento da hanseníase ofertadas no cenário da atenção básica, e implementadas pelos profissionais enfermeiros possuem um alto grau de relevância para controle da doença no território. Dado que, sua atuação objetiva uma assistência qualificada englobando o indivíduo em sua integralidade, a fim de impedir que a doença ocasione incapacidades físicas, que dificultem a socialização do portador de hanseníase em seu convívio social.

Dessa forma, destaca-se que uma das estratégias relevantes desempenhadas pelo enfermeiro compreende a construção da confiança, motivação e compromisso durante a consulta de enfermagem, onde são atribuídas ações responsáveis que integram também a participação conjunta com o paciente visando atenuar as chances de abandono ao tratamento e servindo de meio para explanação acerca da temática auxiliando na colaboração ao enfrentamento da doença no Brasil.

O estudo traz consigo contribuições para a prática de enfermagem que constituem em manter a vigilância epidemiológica principalmente nas regiões com maior incidência, visto que, em países endêmicos, como o caso do Brasil, é percebido que a hanseníase está fortemente ligada aos determinantes e condicionantes de saúde. Além de, demonstrar que há necessidade de uma abordagem mais ampla com a população conduzida pelo profissional enfermeiro no contexto da atenção básica, fazendo uso da educação em saúde em uma 
linguagem facilitada e efetiva, contribuindo para maior afetividade entre a população e o profissional de modo a evitar que abandonem o tratamento, uma vez diagnosticados.

\section{CONFLITOS DE INTERESSES}

Os autores declaram que não há conflitos de interesse. Todos os autores estão cientes da submissão do artigo.

\section{REFERÊNCIAS}

Araújo, K. A., Leano, H., Rodrigues, R. N., Bueno, I. C., \& Lana, F. C. (2017). Tendência de indicadores epidemiológicos da hanseníase em um estado endêmico. Revista da Rede de Enfermagem do Nordeste. 18(6), 771-778.

Araújo, K. M., Gomes, L. C. F., \& Lana, F. C. (2020). Análise espacial do risco de adoecimento da hanseníase em um estado do nordeste brasileiro. Revista Babiana de Enfermagem. 34(37902), 1-11.

Araújo, S. M., \& Silva, L. N. (2019). Vulnerabilidade em casos de hanseníase na atenção primária à saúde. Revista Cientifica da Escola Estadual de Saúde Pública de Goiás. 5(3), 38-50.

Brasil. (2021). Boletim epidemiológico de Hanseníase 2021. Secretaria de Vigilância em Saúde. Departamento de Doenças de Condições Crônicas e Infecções Sexualmente Transmissíveis. Brasília: Ministério da Saúde.

Carvalho, L. K., Tapety, F. I., Valle, A. R., Alves, E. I., Almeida, C. A., Souza, I. B., Silva, A. A., \& Lago, E. C (2018). Capacitação de enfermeiros na Estratégia Saúde da Família: análise do processo de educação permanente para o Sistema Único de Saúde. Revista Nursing: Saúde da Família. 21(247), 2506-2512.

Cavalcante, M., Lorocca, L., \& Chaves, M. (2020). Múltiplas dimensões da gestão do cuidado à hanseníase e os desafios para a eliminação. Revista da Escola de Enfermagem da USP. 54(e03649), 1-8.

Cordeiro, L., \& Soares, C. B. (2019). Revisão de escopo: potencialidades para a síntese de metodologias utilizadas em pesquisa primária qualitativa. Boletim do Instituto de Saúde. 2(20), 37-43.

Cortela, D. C., Ferreira, S. M., Virmond, M. C., Mieras, L., Steinmann, P., Ignotti, E., \& Cavaliero, A. (2020). Aceitabilidade da quimioprofilaxia em área endêmica para hansneíase: projeto PEP-Hans Brasil. CSP. 36(3), 112.

Costa, A. K., Pfrimer, I. A., Menezes, L. B., \& Filho, J. R. (2019). Aspectos clínicos e epidemiológicos da hanseníase. Revista de Enfermagem UFPE on line. 13(1), 353-62.

Freitas, B. H., Silva, F. B., Silva, K. F., Santos, H. C., \& Silva, S. E. (2019). Percepção de adolescentes sobre hanseníase. Revista de Enfermagem UFPE on line. 13(2), 292-297.

Façanha, A. T. F., Conceição, H. N., Oliveira, M. R., Borges, L. V., Pereira, B. M., Moura, L. R., Chaves, T. S., Lima, D. L., \& Câmara, J. T. (2020). Análise das incapacidades físicas por hanseníase em uma cidade do interior do Maranhão, Brasil. Research, Society and Development. 9(2), 1-19.

Gomes, A. Q., Paiva, A. C. B., Cardoso, C. C., Azevedo, C. M., Baia, D. F., Tavares, I. S., Pereira, I. J., Silva, J. M., Barros, K. B., Lopes, L. S., Sarges, M. R., Barreiros, M. P., Soares, M. K., Silva, P. L. (2021). A hanseníase na atenção primária à saúde: Atividades educativas em saúde e seu papel preventivo. Research, Society and Development. 10(7), 1-8. 
Mascarenhas, J. M., Alves, S. P., Souza, M. S., \& Neto, A. M. (2021). A importância das ações realizadas pelo enfermeiro no controle da hanseníase: revisão integrativa. Revista de Casos e Consultoria. 12(1), 1-16.

Monteiro, B. R., Ataíde, C. A., Silva, C. J., Neres, J. N., Medeiros, E. R., \& Simpson, C. A. (2018). Educação em saúde para hanseníase: experiência da enfermagem. Revista Saúde (Santa Maria). 44(1), 1-5.

Morais, J. R., \& Furtado, E. Z. L. (2018). Grau de incapacidade Física de pacientes com hanseníase. Revista de Enfermagem UFPE on line. 12(6), 1625-32.

Moreira, A. S., Rocha, L. G., Santos, V. Y., Santos, I. V., Rocha, I. M., \& Tavares, C. M. (2021). Atuação dos enfermeiros nas ações de controle da hanseníase na atenção primária à saúde: revisão integrativa. Diversitas Journal. 6(4), 3949-3966.

Oliveira, A. E., Araújo, K. M., Queiroga, R. P., Bezerra, L. L., \& Chaves., A. E. (2020a). Análise epidemiológica da hanseníase por sexo na Paraíba. Research, Society and Development. 9(8), 1-15.

Oliveira, M. D., Lima, J. O., Júnior, F. G., Garcia, T. R., \& Bachion, M. M. (2020b). Diagnósticos de enfermagem em pessoas com hanseníase: aproximação entre teoria de Ordem e a CIPE. Revista eletrônica de enfermagem. 22(63602), 1-9.

Organização Mundial da Saúde (OMS). (2020). Weekly epidemiological record. Biol Proc Online: file:///C:/Users/Administrador/Downloads/WER9536-eng-fre\%20(1).pdf

Palmeira, I. P., Nunes, M. J., Gaia, E. S., Ferreira, A. M., \& Boulhosa, M. (2020). Percepção de pacientes com hanseníase sobre suas necessidades humanas básicas alteradas: indícios para o autocuidado. Revista de Pesquisa Cuidado é Fundamental Online. 12(7069), 319-325.

Joanna Briggs Institute (JBI). (2020). Chapter 11: Scoping Reviews. Biol Proc Online: https://jbi-globalwiki.refined.site/space/MANUAL/3283910770/Chapter+11\%3A+Scoping+reviews

Pinheiro, M. G., Miranda, F. A., Simpson, C. A., Carvalho, F. P., Ataíde, K. A., \& Lira, A. L. (2017). Compreendendo a "alta em hanseníase”: uma análise de conceito. Revista Gaúcha de Enfermagem. 38(4), 1-8.

Ramos, J. S., Costa, L. R. \& Santos, W. L. (2019). Dificuldades da enfermagem no manejo da hanseníase na atenção primária. Revista JRG de Estudos Acadêmicos. 2(5), 125-147.

Ribeiro, M. D., Castillo, I. S., Silva, J. C. A., \& Oliveira, S. B. (2017). A visão do profissional enfermeiro sobre o tratamento da hanseníase na atenção básica. Revista Brasileira em Promoção da Saúde. 20(2), 221-228.

Sousa, G. S., Silva, R. F. \& Xavier, M. B. (2017). Atributos da Atenção Primária em Saúde no controle da hanseníase: ótica do enfermeiro. Revista Baiana de enfermagem. 31(1), 1-10.

Souza, C. D., Magalhães, M. A. \& Luna, C. F. (2020). Hanseníase e carência social: definição de áreas prioritárias em estado endêmico do Nordeste brasileiro. Revista brasileira de epidemiologia. 23(2), 1-14.

Souza, R. G., Lanza, F. M. \& Souza, R. S. (2018). Sensibilização dos Agentes Comunitários de Saúde para a atuação nas ações prevenção e controle da hanseníase: relato de experiência. HU Revista. 44(3), 411-415.

Vieira, N. F., Martinez-Rieira, J. R. \& Lana, F. C. (2020). Qualidade da atenção primária e os efeitos em indicadores de monitoramento da hanseníase. Revista Brasileira de Enfermagem. 73(4), 1-8.

World Health Organization. MDT: Managing Irregular Treatment Faq. (2019). Geneva: WHO. 


\section{Contribuições dos autores}

Todos os autores contribuíram com a coleta dos dados, escrita e revisão do trabalho. 